\title{
Effect of Ball-Milling Conditions on Thermoelectric Properties of Polycrystalline CuGaTe 2
}

\author{
Masaya Kumagai ${ }^{1, * 1, * 2}$, Ken Kurosaki ${ }^{1, * 2}$, Yuji Ohishi ${ }^{1}$, \\ Hiroaki Muta ${ }^{1}$ and Shinsuke Yamanaka ${ }^{1,2}$ \\ ${ }^{1}$ Graduate School of Engineering, Osaka University, Suita 565-0871, Japan \\ ${ }^{2}$ Research Institute of Nuclear Engineering, University of Fukui, Tsuruga 914-0055, Japan
}

$\mathrm{CuGaTe}_{2}$ has a large thermoelectric (TE) figure of merit $(Z T)$ at high temperatures, i.e., $Z T=1.4$ at $950 \mathrm{~K}$. However, at lower temperatures, the $Z T$ values are smaller. One reason for this is that $\mathrm{CuGaTe}_{2}$ has a high lattice thermal conductivity $\left(\kappa_{\text {lat }}\right)$ at lower temperatures. One known method for reducing $\kappa_{\text {lat }}$ is manipulating the microstructure of the bulk material. Therefore, herein, the grain-size effect on the TE properties of $\mathrm{CuGaTe}_{2}$ bulk samples produced by ball milling and hot pressing was investigated. Although $\kappa_{\text {lat }}$ decreased with grain size, a simultaneous decrease in the Seebeck coefficient occurred, leading to no overall improvement in ZT. [doi:10.2320/matertrans.E-M2014821]

(Received October 16, 2013; Accepted March 14, 2014; Published May 2, 2014)

Keywords: thermoelectric, $\mathrm{CuGaTe}$, chalcopyrite, thermal conductivity, electrical resistivity, Seebeck coefficient

\section{Introduction}

Thermoelectric (TE) materials can convert waste heat into electric power; this method is an attractive method to reduce greenhouse gas emissions. ${ }^{1,2)}$ The effectiveness of a TE material is determined by the dimensionless figure of merit, $Z T=S^{2} T / \rho / \kappa$, where $S, \rho, T$, and $\kappa$ are, respectively, the Seebeck coefficient, electrical resistivity, absolute temperature, and total thermal conductivity. Also, $\kappa=\kappa_{\text {lat }}+\kappa_{\mathrm{el}}$, where $\kappa_{\text {lat }}$ and $\kappa_{\mathrm{el}}$ are the lattice and electronic thermal conductivities, respectively. Because $S, \rho$, and $\kappa_{\mathrm{el}}$ are interrelated in bulk materials, it is difficult to control them independently. Consequently, it is perhaps easier to reduce $\kappa_{\text {lat }}$ for increasing $Z T$. The $Z T$ values of the materials used in current TE devices are approximately unity.

For developing a TE power generator for the consumer market such as for the automotive industry, one constraint is that the device efficiency should be of at least $15 \%$, corresponding to a $Z T$ value of 1.5 or more. Although several bulk materials with $Z T$ greater than 1.5 have been developed, ${ }^{3-9)}$ many are based on PbTe-type alloys such as $\mathrm{AgPb}_{m} \mathrm{SbTe}_{m+2}$ (LAST- $\left.m\right)$, ${ }^{5}$ AgTe-PbTe with La, $\left.{ }^{6}\right) \mathrm{PbTe}-$ $\mathrm{SrTe},{ }^{7,8)} \mathrm{NaPb}_{m} \mathrm{SbTe}_{2+m}(\mathrm{SALT}-m),{ }^{9}$ and $\mathrm{PbTe}_{1-x} \mathrm{Se}_{x}{ }^{10}{ }^{10}$

Herein, we focus on $\mathrm{CuGaTe}_{2}$, which has a chalcopyrite structure and a relatively large $Z T$ of up to 1.4 at $950 \mathrm{~K} .^{11)}$ Although $\mathrm{CuGaTe}_{2}$ exhibits a large $Z T$ value at elevated temperatures, at lower temperatures the $Z T$ value is much smaller. One reason for this is that $\mathrm{CuGaTe}_{2}$ has a high $\kappa_{\text {lat }}$ at lower temperatures. Because $\kappa_{\text {lat }}$ is dependent on the microstructure of bulk material, the grain-size effect on the TE properties of $\mathrm{CuGaTe}_{2}$ bulk samples produced by ball milling and hot pressing was investigated in the present study. Ball milling is generally used to control the grain size of a sample by taking into consideration several conditions (such as milling time, the diameter of the ball, revolutions per minute, ball to powder ratio, and the type of ball). In this

\footnotetext{
${ }^{* 1}$ Graduate Student, Osaka University

${ }^{* 2}$ Corresponding author, E-mail: kumagai-m@ms.see.eng.osaka-u.ac.jp, kurosaki@see.eng.osaka-u.ac.jp
}

study, we attempt to control the grain size by changing the milling time only.

\section{Experiment}

$\mathrm{CuGaTe}_{2}$ was prepared by a direct reaction of pieces of $\mathrm{Cu}$ (99.99\%), Ga (99.99\%), and Te (99.999\%) in a sealed silica tube. The ingots obtained after melting were crushed into powder by hand, followed by ball milling. A planetary ball mill (Fritsch Japan Co., Ltd., PULVERISETTE7) with a WC vial volume of $45 \times 10^{3} \mathrm{~mm}^{3}$ and WC balls with a diameter of $1.6 \mathrm{~mm}$ was used. The ball to powder ratio was set to 2.6:1. Milling was performed at $300 \mathrm{rpm}$ under $\mathrm{Ar}$ atmosphere. To control the grain size, the ball-milling time was set to 0,1 , or $3 \mathrm{~h}$. Following ball milling, each powder was hotpressed in a graphite die using hotpress systems (Motoyama Co., Ltd.) at $823 \mathrm{~K}$ for $3 \mathrm{~h}$ in a flowing $\mathrm{Ar}$ atmosphere. The resulting bulk samples were characterized using powder X-ray diffraction (XRD) with $\mathrm{CuK} \alpha$ radiation (Rigaku Corp., UltimaIV) at room temperature. The microstructure of the samples was examined using field emissionscanning electron microscopy (FE-SEM). JSM-6500F (JEOL Ltd.) microscope was used to obtain SEM images. The values of $\rho$ and $S$ were measured in the temperature range 300$973 \mathrm{~K}$ in a He atmosphere using a commercial measurement system (ULVAC, ZEM-1). The Hall coefficient $\left(R_{\mathrm{H}}\right)$ was measured at room temperature by the van der Pauw method under an applied magnetic field of $0.5 \mathrm{~T}$ using the Hall effect/ resistivity measurement system (TOYO Corp., ResiTest8300). The values of Hall carrier concentration $\left(n_{\mathrm{H}}\right)$ and Hall mobility $\left(\mu_{\mathrm{H}}\right)$ were calculated from $R_{\mathrm{H}}$ based on the assumptions of a single-band model and a Hall factor of 1 , i.e., $n_{\mathrm{H}}=1 /\left(e R_{\mathrm{H}}\right)$ and $\mu_{\mathrm{H}}=R_{\mathrm{H}} / \rho$, where $e$ is the elementary electric charge. The parameter $\kappa$ was evaluated from thermal diffusivity $(\alpha)$, heat capacity $\left(C_{\mathrm{p}}\right)$, and density $(d)$ using the relationship $\kappa=\alpha C_{\mathrm{p}} d$. The value of $\alpha$ was measured in vacuum from room temperature to $973 \mathrm{~K}$ using a laser flash thermal constant analyzer (ULVAC, TC-7000). In this study, $C_{\mathrm{p}}$ was estimated from $C_{\mathrm{p}}=3 n R+9 B_{\mathrm{T}} T V_{\mathrm{m}} \alpha_{\mathrm{e}}^{2}$, where $n$ is the number of atoms per formula unit and $R$ is the gas 
Table 1 Ball-milling conditions, estimated grain size, sample bulk density, Hall carrier concentration $\left(n_{\mathrm{H}}\right)$, and Hall mobility $\left(\mu_{\mathrm{H}}\right)$ for $\mathrm{CuGaTe}_{2}$ samples. Density, $n_{\mathrm{H}}$, and $\mu_{\mathrm{H}}$ were evaluated at room temperature.

\begin{tabular}{ccccc}
\hline $\begin{array}{c}\text { Ball-milling } \\
\text { condition }\end{array}$ & $\begin{array}{c}\text { Grain size } \\
\text { estimated from } \\
\text { FE-SEM } \\
\text { images/ } \mu \mathrm{m}\end{array}$ & $\begin{array}{c}\text { Density/ } \\
\% \text { T.D. }\end{array}$ & $\begin{array}{c}n_{\mathrm{H}} / \\
10^{24} \mathrm{~m}^{-3}\end{array}$ & $\begin{array}{c}\mu_{\mathrm{H}} / R_{\mathrm{H}} / \\
10^{-4} \mathrm{~m}^{2} \mathrm{~V}^{-1} \mathrm{~s}^{-1}\end{array}$ \\
\hline $\begin{array}{c}\text { Not ball } \\
\text { milled }\end{array}$ & $\sim 50$ & 99.7 & 3.35 & 67.8 \\
\hline $300 \mathrm{rpm}, 1 \mathrm{~h}$ & $\sim 5$ & 99.9 & 13.2 & 34.9 \\
\hline $300 \mathrm{rpm}, 3 \mathrm{~h}$ & $\sim 0.5$ & 95.7 & 45.6 & 0.47 \\
\hline
\end{tabular}

constant, $B_{\mathrm{T}}$ is the isothermal bulk modulus, $V_{\mathrm{m}}$ is the specific volume, and $\alpha_{\mathrm{e}}$ is the average coefficient of linear thermal expansion. $B_{\mathrm{T}}$ was estimated from $B_{\mathrm{T}}=B_{\mathrm{s}} /\left(1+\alpha_{\mathrm{e}} \gamma T\right), B_{\mathrm{s}}$ being the adiabatic bulk modulus and $\gamma$ the Grüneisen parameter. ${ }^{12)}$ In this study, the values of $B_{\mathrm{s}}$ and $\gamma$ of $\mathrm{CuGaTe}_{2}$ were $51.5 \mathrm{GPa}$ and 1 , respectively. ${ }^{11)}$ The density of the hotpressed samples was calculated from the measured weight and dimensions.

\section{Results and Discussion}

The grain size in the $\mathrm{CuGaTe}_{2}$ powder was controlled by changing the ball-milling time. The FE-SEM images of the fractured surface of bulk samples produced by hot pressing are shown in Figs. 1(a), 1(b), and 1(c) for ball-milling times of 0,1 , and $3 \mathrm{~h}$, respectively. The estimated grain size of the samples using the ball-milling times of 0,1 and $3 \mathrm{~h}$ from the FE-SEM images were $\sim 50,5$, and $0.5 \mu \mathrm{m}$ respectively. These results are listed in Table 1.

Figure 2 shows the powder XRD patterns for the hotpressed samples, together with the peak positions for $\mathrm{CuGaTe}_{2}$ reported in Ref. 13). All the peaks in the XRD patterns can be indexed in terms of the chalcopyrite structure with the space group $I \overline{4} 2 d$. The purities of the source materials were $99.99,99.99$, and $99.999 \%$ for $\mathrm{Cu}, \mathrm{Ga}$, and Te, respectively. Furthermore, all the peaks in the XRD patterns of the samples agreed well with the data presented in the literature, as shown in Fig. 2. Therefore, the total amount of impurities is less than $1 \%$, which leads to no significant influence on the TE properties of the samples. Moreover, it can be concluded that single-phase samples of $\mathrm{CuGaTe}_{2}$ were obtained in the present study. The lattice parameters calculated from the XRD patterns are shown in Fig. 2, and are slightly different from the reported values. ${ }^{13)}$

The ball-milling conditions, estimated grain size, sample bulk density, Hall coefficient $\left(R_{\mathrm{H}}\right)$, Hall carrier concentration $\left(n_{\mathrm{H}}\right)$, and Hall mobility $\left(\mu_{\mathrm{H}}\right)$ for the $\mathrm{CuGaTe}_{2}$ samples are summarized in Table 1 . The values of density, $R_{\mathrm{H}}, n_{\mathrm{H}}$, and $\mu_{\mathrm{H}}$ were evaluated at room temperature. The relative densities of the two samples with ball-milling times of 0 and $1 \mathrm{~h}$ were $\sim 100 \%$ of the theoretical density, while that of the sample with ball milling time of $3 \mathrm{~h}$ was $\sim 96 \%$. The $R_{\mathrm{H}}$ values for all the samples were positive, signifying that most of the charge carriers were holes. The $n_{\mathrm{H}}$ value increased with ball-milling time. It is considered that the mechanical energy associated with ball milling causes the $\mathrm{Cu}$ atoms to move to interstitial sites, leaving $\mathrm{Cu}$ vacancies that act as acceptors. ${ }^{14,15)}$ In
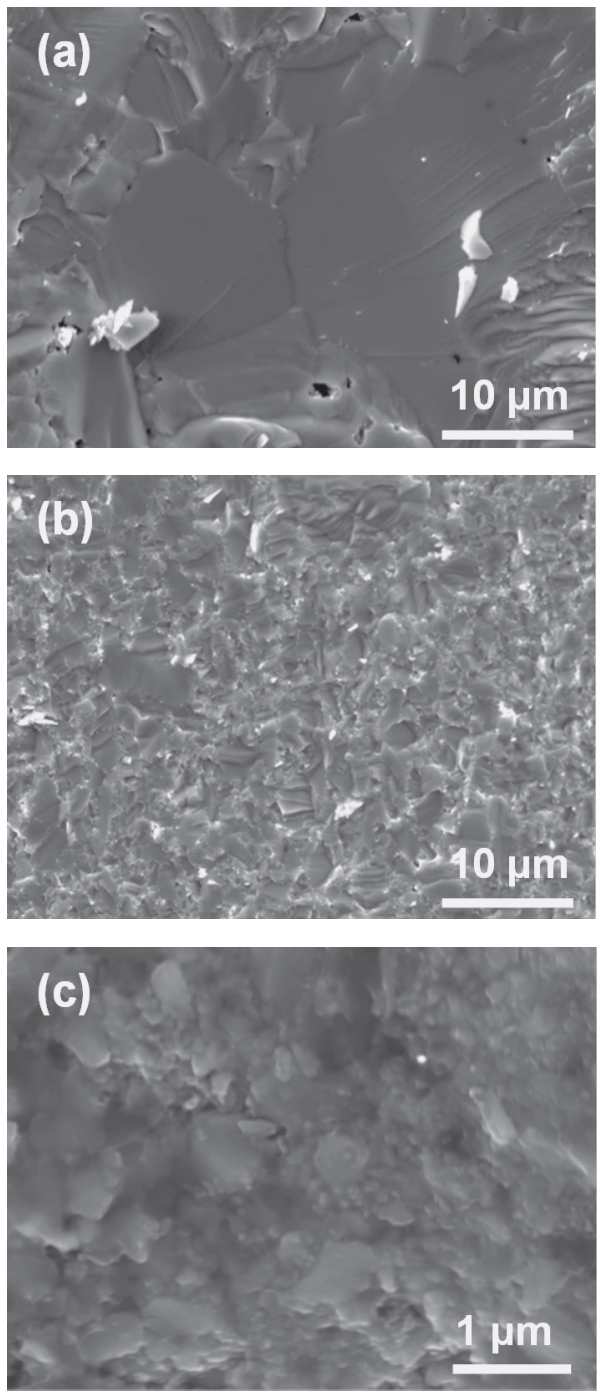

Fig. 1 FE-SEM images of $\mathrm{CuGaTe}_{2}$ samples after hot pressing; (a) without ball milling, (b) ball milled (300 rpm, $1 \mathrm{~h})$, and (c) ball milled (300 rpm, $3 \mathrm{~h})$.

addition, because the most common intrinsic defects in $\mathrm{CuGaTe}_{2}$ are Te vacancies, $\mathrm{Cu}$ atoms vacating the $\mathrm{Cu}$ sites tend to occupy the Te sites. Therefore, it is likely that the hole concentration increases with ball-milling time. However, those defects could not be confirmed from the variation of lattice parameters, as shown in Fig. 2. Additionally, a favorable result was not achieved from Rietveld analysis based on the XRD patterns. On the other hand, the hole mobility $\mu_{\mathrm{H}}$ was found to decrease with increasing ball- 
milling time; this is thought to be due to an increase in the number of grain boundaries and point defects (e.g., $\mathrm{Cu}$ vacancies), which are responsible for carrier scattering.

The temperature dependence of the TE properties of the samples is shown in Fig. 3. All $S$ values are positive, indicating that the majority of the charge carriers are holes.

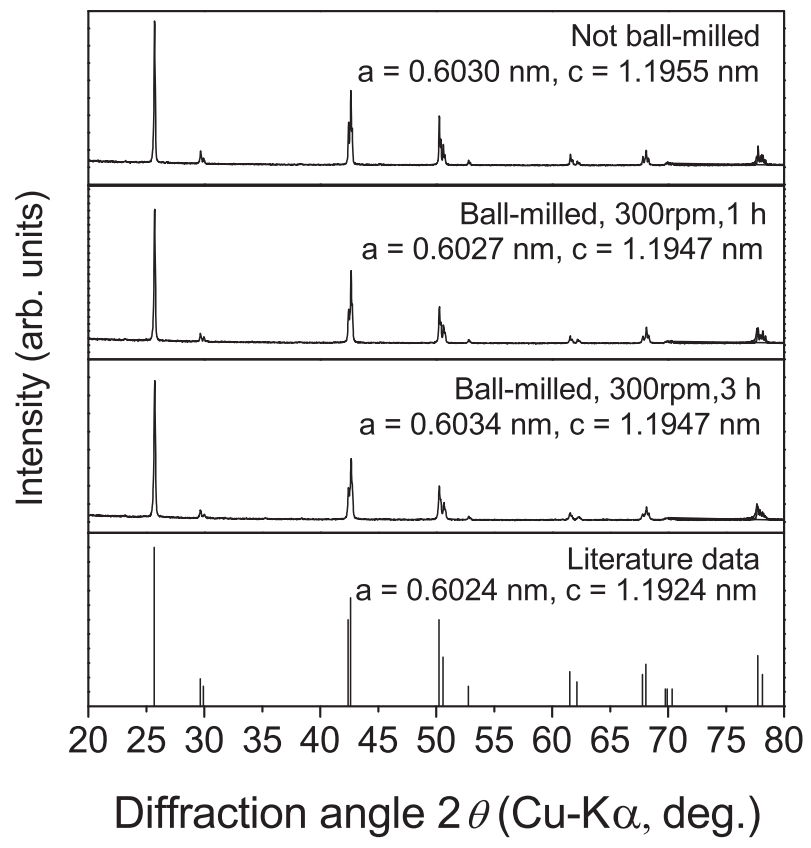

Fig. 2 Powder XRD patterns for $\mathrm{CuGaTe}_{2}$ samples after hot pressing, together with literature data.
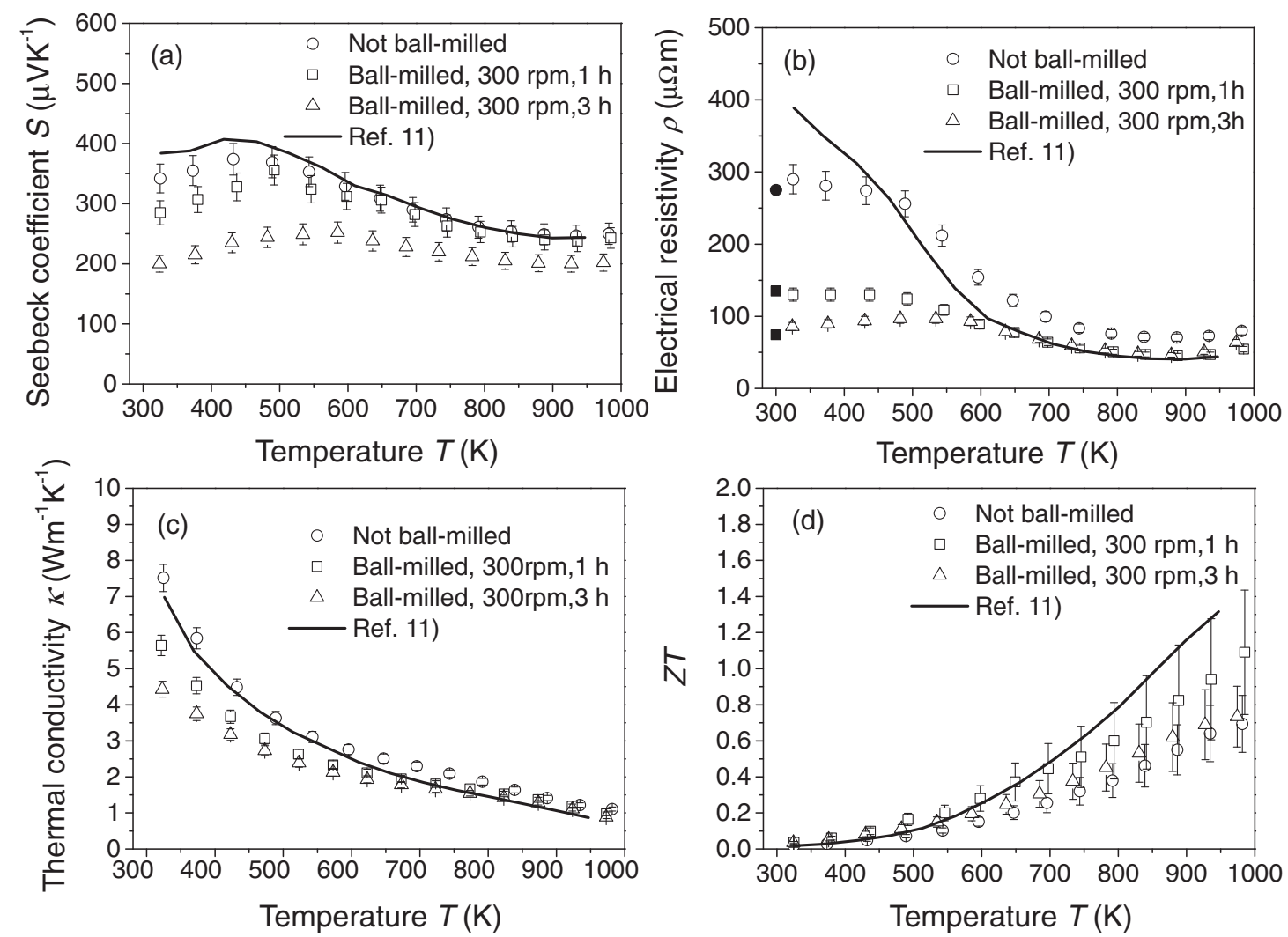

This is consistent with the results of the Hall effect measurements. For all the samples, $S$ first increased slightly up to $\sim 500 \mathrm{~K}$, and then decreased with increasing temperature. Because the three samples indicated different density values, the $\kappa$ and $\rho$ values of the samples were compensated to those for fully dense $\mathrm{CuGaTe}_{2}$ by Maxwell-Eucken's equation. $^{16)}$

$$
\kappa_{\mathrm{p}}=\kappa(1-p) /(1+\beta p)
$$

where $p$ is the porosity, $\beta$ is the constant determined by the conditions of the pores (in the present case, $\beta=0.5$ ), $\kappa_{\mathrm{p}}$ is the thermal conductivity with porosity $p$, and $\kappa$ is the thermal conductivity of a fully dense sample. The electrical resistivity was also compensated by the same method, wherein $\kappa$ was replaced by $1 / \rho$. The compensated results are plotted in Figs. 3(a) and 3(c). The $\rho$ values for all the samples were almost independent of temperature up to $\sim 500 \mathrm{~K}$, and then decreased with increasing temperature. These results imply that below $500 \mathrm{~K}$, electron excitation arises from impurity levels; however, at high temperature, the influence of the impurities becomes negligible. The decrease in both the $\rho$ and absolute values of $S$ above $500 \mathrm{~K}$ is believed to be due to the thermal excitation of holes. In addition, both the $\rho$ and absolute values of $S$ decrease with increasing ball-milling time because of the increase in the hole concentration (Figs. 3(a) and (b)).

The decrease in $\kappa$ with increasing temperature roughly follows the $T^{-1}$ relationship (Fig. 3(c)). This means that thermal conduction in $\mathrm{CuGaTe}_{2}$ is mainly determined by the typical lattice contribution dominated by Umklapp scattering.

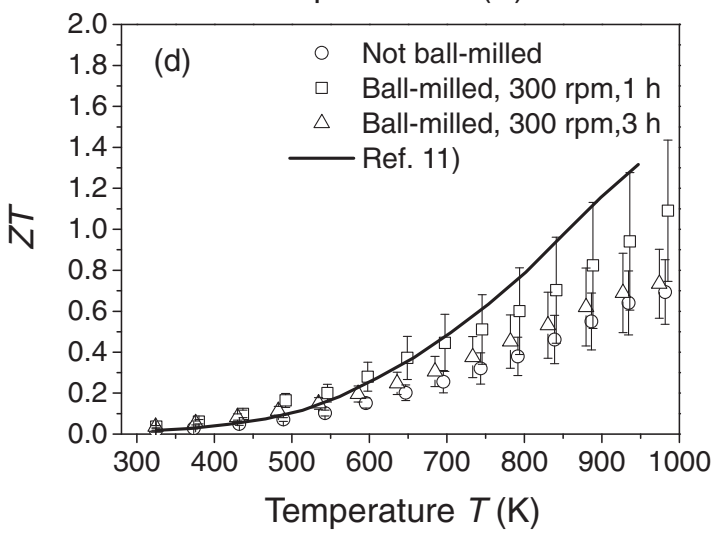

Fig. 3 Temperature dependence of thermoelectric properties of $\mathrm{CuGaTe}_{2}$ samples; (a) Seebeck coefficient $S$, (b) electrical resistivity $\rho$, (c) thermal conductivity $\kappa$, and (d) dimensionless figure of merit ZT. Filled symbols in Fig. 3(b) represent results obtained from Hall measurements. 
To evaluate $\kappa_{\text {lat }}$, the electronic contribution $\left(\kappa_{\mathrm{el}}=L T \rho^{-1}\right.$, where $L=1.5 \times 10^{-8} \mathrm{~W} \Omega \mathrm{K}^{-2}$ for nondegenerate materials) was subtracted from the measured $\kappa$, i.e., $\kappa_{\text {lat }}=\kappa-L T \rho^{-1}$. At room temperature, $\kappa_{\mathrm{el}}$ can be considered to be negligible. It was also found that $\kappa$ decreased with increasing ballmilling time at room temperature. This can be attributed to an increase both in grain boundary scattering due to the decreased grain size and point defect scattering due to the $\mathrm{Cu}$ vacancies.

The maximum $Z T$ for the samples used in the present study was $\sim 1.2$ at $973 \mathrm{~K}$ (Fig. 3(d)). This value is slightly lower than the maximum value reported. ${ }^{11)}$ It was also found that $Z T$ decreased with increasing ball-milling time, and this can be primarily attributed to the decrease in the absolute value of $S$.

The reproducibility of the experimental results is very important. In the present case, we measured the TE properties a few times for each sample and confirmed that the results were consistent with each other within the experimental errors for each measurement, i.e., approximately $\pm 7 \%$ for $\rho$, $\pm 7 \%$ for $S$, and $\pm 5 \%$ for $\alpha$. On the basis of the errors for each measurement, the errors of $Z T$ for the three samples with ballmilling times of 0,1 , and $2 \mathrm{~h}$ were estimated to be 26,37 , and $31 \%$, respectively.

\section{Summary}

We attempted to control the microstructure of $\mathrm{CuGaTe}_{2}$ bulk samples produced by ball milling of $\mathrm{CuGaTe}_{2}$ powders followed by hot pressing. The grain size was found to decrease from $\sim 50$ to $0.5 \mu \mathrm{m}$ with increasing ball-milling time. In the final hot-pressed samples, it was considered that increasing the ball-milling time increased the number of grain boundaries and $\mathrm{Cu}$ vacancies. This led to a greater hole concentration and a decrease in mobility and lattice thermal conductivity $\kappa_{\text {lat. }}$. Consequently, $Z T$ decreased with increasing ball-milling time. In the present study, the maximum $Z T$ value was $\sim 1.2$ at $973 \mathrm{~K}$, and was obtained for the sample milled for $1 \mathrm{~h}$. However, because a reduction in $\kappa_{\text {lat }}$ also occurred simultaneously, it is possible that $Z T$ can be improved by adjusting the carrier concentration through doping.

\section{Acknowledgements}

This work was supported in part by a Grant-in-Aid for Scientific Research (No. 23686091) from the Ministry of Education, Culture, Sports, Science and Technology, Japan.

\section{REFERENCES}

1) T. M. Tritt and M. A. Subramanian: MRS Bull. 31 (2006) 188-198.

2) G. Homm and P. J. Klar: Phys. Status Solidi RRL 5 (2011) 324-331.

3) X. Shi, J. Yang, J. R. Salvador, M. Chi, J. Y. Cho, H. Wng, S. Bai, J. Yang, W. Zhang and L. Chen: J. Am. Chem. Soc. 133 (2011) 78377846.

4) S. H. Yang, T. J. Zhu, T. Sun, J. He, S. N. Zhang and X. B. Zhao: Nanotechnology 19 (2008) 245707.

5) K. F. Hsu, S. Loo, F. Guo, W. Chen, J. S. Dyck, C. Uher, T. Hogan, E. K. Polychroniadis and M. G. Kanatzidis: Science 303 (2004) 818821.

6) Y. Pei, J. Lensch-Falk, E. S. Toberer, D. L. Medli and G. J. Snyder: Adv. Funct. Mater. 21 (2011) 241-249.

7) K. Biswas, J. He, Q. Zhang, G. Wang, C. Uher, V. P. Dravid and G. Kanatzidis: Nat. Chem. 3 (2011) 160-166.

8) K. Biswas, J. He, I. D. Blum, C. I. Wu, T. P. Hogan, D. N. Seidman, V. P. Dravid and M. G. Kanatzidis: Nature 489 (2012) 414-418.

9) P. F. P. Poudeu, J. D. Angelo, A. D. Downey, J. L. Short, T. P. Hogan and M. G. Kanatzidis: Angew. Chem. Int. Ed. 45 (2006) 3835-3839.

10) Y. Pei, X. Shi, A. Lalonde, H. Wang, L. Chen and G. J. Snyder: Nature 473 (2011) 66-69.

11) T. Plirdpring, K. Kurosaki, A. Kosuga, T. Day, A. Firdosy, V. Ravi, G. J. Snyder, A. Harnwunggmoung, T. Sugaharam, Y. Ohishi, H. Muta and S. Yamanaka: Adv. Mater. 24 (2012) 3622-3626.

12) G. A. Slack and V. G. Tsoukala: J. Appl. Phys. 76 (1994) 1665-1671.

13) I. V. Bodnar and N. S. Orlova: Cryst. Res. Technol. 21 (1986) 10911096.

14) G. Masse and E. Redjai: J. Phys. Chem. Solids 47 (1986) 99-104.

15) H. Neumann: Cryst. Res. Technol. 18 (1983) 901-906.

16) A. Eucken: Forschung 11 (1940) 6-20. 